\title{
Active Control of Surface Plasmon Waveguides with a Phase Change Material
}

\author{
Miquel Rudé, ${ }^{*}{ }^{\dagger}$ Robert E. Simpson, ${ }^{\ddagger}$ Romain Quidant, ${ }^{\dagger, \perp}$ Valerio Pruneri, ${ }^{\dagger, \perp}$ and Jan Renger ${ }^{\S}$ \\ ${ }^{\dagger}$ ICFO - The Institute of Photonic Sciences, Mediterranean Technology Park, Avenida Carl Friedrich Gauss 3, 08860 Castelldefels \\ (Barcelona), Spain \\ ${ }^{\ddagger}$ Engineering Product Development, Singapore University of Technology and Design, 8 Somapah Road, Singapore 487372, Singapore \\ ${ }^{\perp}$ ICREA - Institució Catalana de Recerca i Estudis Avançats, Passeig Lluís Companys 23, 08010 Barcelona, Spain \\ ${ }^{\S}$ Photonics Laboratory, ETH Zürich, Hönggerbergring 64, 8093 Zürich, Switzerland
}

\section{Supporting Information}

ABSTRACT: The ability to manipulate light propagation at the nanoscale is of vital importance for future integrated photonic circuits. In this work we exploit the high contrast in the optical properties of the phase change material $\mathrm{Ge}_{2} \mathrm{Sb}_{2} \mathrm{Te}_{5}$ to control the propagation of surface plasmon polaritons at a $\mathrm{Au} / \mathrm{SiO}_{2}$ interface. Using grating couplers, normally incident light at $\lambda=1.55 \mu \mathrm{m}$ is converted into propagating surface plasmons on a Au waveguide. Single laser pulses $(\lambda=975 \mathrm{~nm})$ are applied to a thin film of $\mathrm{Ge}_{2} \mathrm{Sb}_{2} \mathrm{Te}_{5}$ placed on top of the device, which, upon transition from its amorphous to crystalline structural phase, dramatically increases both its refractive index and absorption coefficient, thus inhibiting propagation of the plasmonic mode. This effect is investigated for different interaction lengths between the phase change material and the Au waveguide, and contrast values in the transmitted intensity up to several tens of percents are demonstrated.

KEYWORDS: phase change materials, surface plasmon, nanophotonics, nonvolatile, chalcogenides

$I^{n}$ $\mathrm{n}$ recent years there has been an ongoing effort to develop novel photonic circuits with high processing speed and robustness against fabrication tolerances. Optical data communication already outperforms their electronic counterparts in terms of speed and transmission losses. However, due to the barrier imposed by the diffraction limit of light, ${ }^{1}$ conventional optical components usually have dimensions larger than the wavelength of light and are quite sensitive to changes in their geometry arising from fabrication tolerances, thus making it difficult to achieve both high field confinement and robustness. Surface plasmon polaritons (SPPs) emerged as a promising candidate for solving these drawbacks of conventional photonic circuits. $^{2-4}$

SPPs are hybrid modes that are bound at the metal-dielectric interface when a light wave is coupled to the oscillation of the free electrons present in the metal. These waves are confined to the interface with an electric field that decays exponentially in both surrounding materials. Thus, the light is strongly confined, which provides a platform to guide it in compact devices ${ }^{5,6}$ that are smaller than conventional optical components, such as electrooptic modulators or optical fibers.

One of the main challenges of plasmonic-based circuits is finding a way to control SPP propagation. This problem has been tackled by changing the optical properties of the surrounding environment, for instance by exploiting the electro-optic effect, ${ }^{7,8}$ using quantum dots, ${ }^{9}$ or by externally pumping photochromic molecules. ${ }^{10}$ However, the majority of such proof-of-principle experiments employ modulator designs that weakly effect the SPP propagation and therefore exhibit a limited SPP modulation depth. More recently, photonic switches and modulators using low-dimensional materials such as graphene have also been demonstrated ${ }^{11}$ that can achieve high modulation frequencies in the $\mathrm{GHz}$ regime but that require the continuous application of electric fields and are more prone to long-term instabilities due to interface effects.

Here, we use the unique features of the prototypical phase change material (PCM) $\mathrm{Ge}_{2} \mathrm{Sb}_{2} \mathrm{Te}_{5}(\mathrm{GST})^{12}$ to perform nonvolatile control of SPP propagation in $\mathrm{Au}$ waveguides. PCMs on the GeTe- $\mathrm{Sb}_{2} \mathrm{Te}_{3}$ pseudobinary tie-line exhibit an extraordinarily large contrast between their two structural phases. The covalently bonded amorphous phase of GST corresponds to a disordered material with short-range atomic order and low electrical conductivity and optical absorption $\left(\tilde{n}_{\text {amorph }}=4.7+0.2 i\right.$ at $\left.\lambda=1.55 \mu \mathrm{m}\right)$. In contrast, the resonantly bonded crystalline phase can be seen as a low-band-gap semiconductor and exhibits an electrical conductivity that is 3 orders of magnitude greater than the amorphous phase and has a larger optical absorption $\left(\tilde{n}_{\text {cryst }}=7+2 i\right.$ at $\left.\lambda=1.55 \mu \mathrm{m}\right){ }^{13-17}$ The large refractive index of the crystalline phase is due to the

Received: February 5, 2015

Published: May 25, 2015 
a)

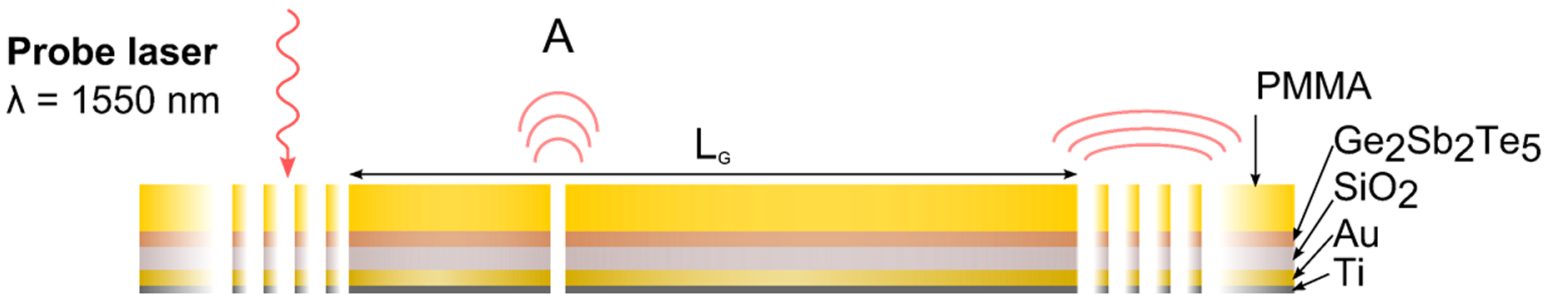

b)
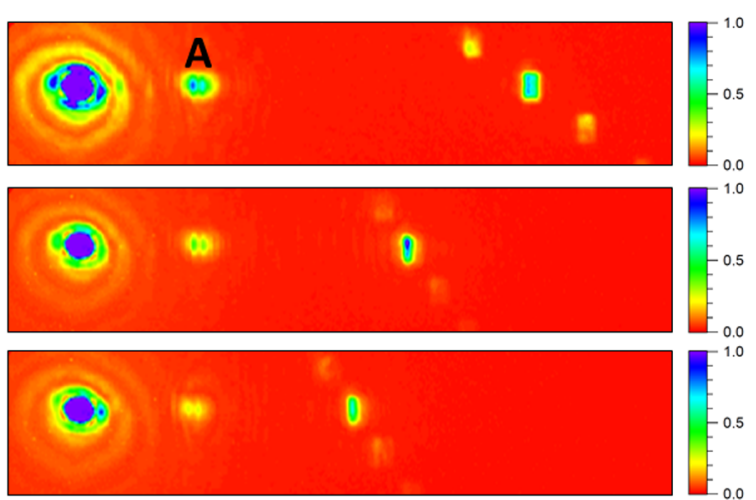

c)

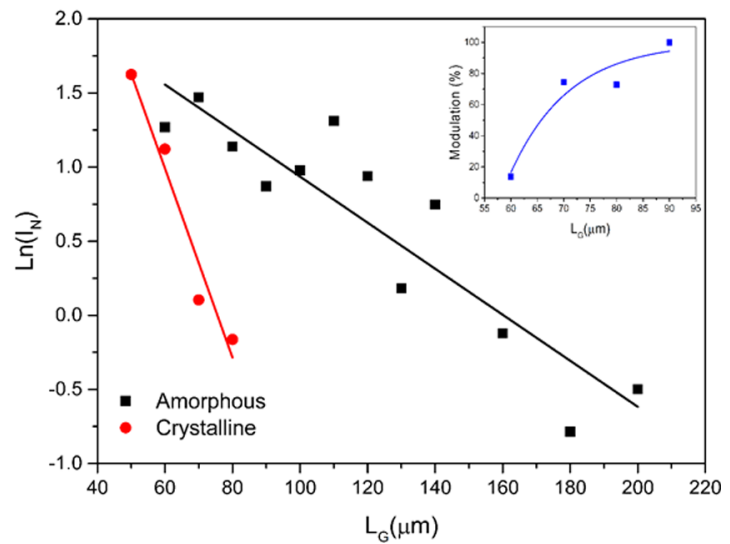

Figure 1. (a) Schematic of the devices used to probe the propagation length of SPPs in Au/PCM hybrid waveguides. (b) Intensity images of three devices with different values of $L_{\mathrm{G}}(160,100$, and $80 \mu \mathrm{m})$ with the GST in the amorphous phase. (c) Logarithm of the normalized intensity at the output port as a function of the distance between gratings with the GST in the amorphous (black squares) and crystalline (red circles) phases. The output value is normalized by the intensity scattered at a single groove (A) placed at a fixed distance from the input grating.

a)

\section{Cross section}

\begin{tabular}{|c|c|c|}
\hline \multirow{3}{*}{$\begin{array}{l}\text { PMMA } 2 \mu \mathrm{m} \rightarrow \\
\text { GST } 80 \mathrm{~nm} \rightarrow\end{array}$} & $\begin{array}{c}\text { Cover layer } \\
\text { Protection layer }\end{array}$ & $\mathrm{Si}_{3} \mathrm{~N}$ \\
\hline & Phase change layer & \\
\hline & Buffer layer & $\leftarrow \mathrm{SiO}_{2} 150 \mathrm{~nm}$ \\
\hline Au $60 \mathrm{~nm}^{\nearrow}$ & $\begin{array}{c}\text { Plasmonic Waveguide } \\
\text { Adhesion layer }\end{array}$ & \\
\hline
\end{tabular}

c)

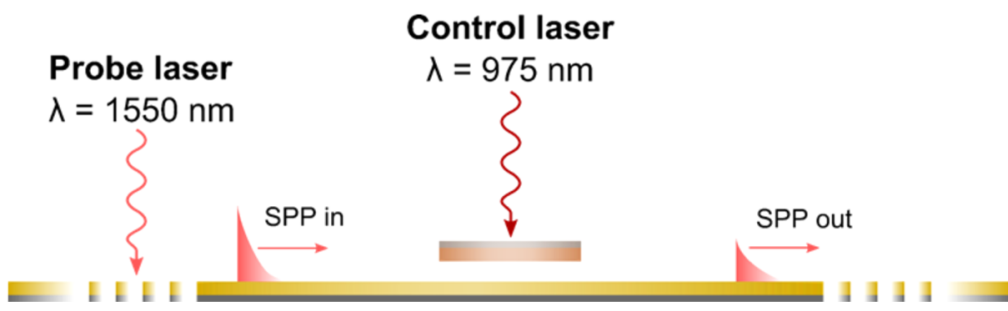

b)

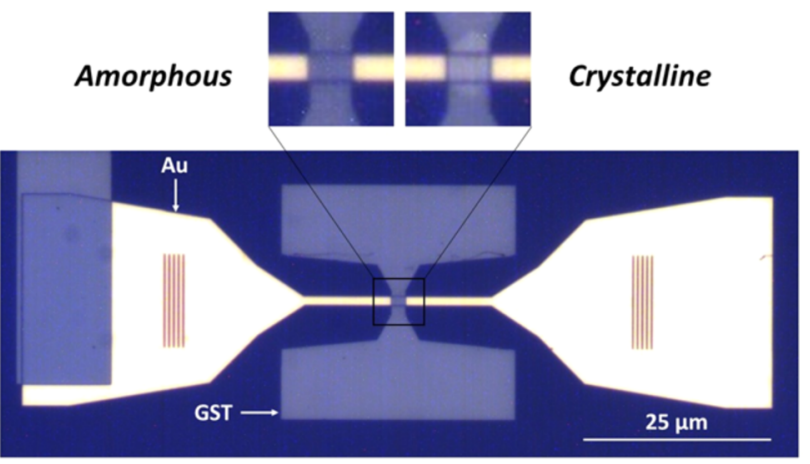

Figure 2. (a) Schematic of the cross section in the interaction region (not to scale). (b) Optical microscope image of the plasmonic waveguide with a GST strip in the middle. The insets show the GST area before and after crystallization using a single laser pulse. (c) Principle of operation of the device. A probe laser at $\lambda=1.55 \mu \mathrm{m}$ is used to convert free space light into propagating SPPs that interact with an $80 \mathrm{~nm}$ GST film. An independent pump laser at $\lambda$ $=975 \mathrm{~nm}$ is used to trigger the amorphous to crystalline phase transition in this film.

existence of resonant bonds, which can be easily polarized by an external electric field. ${ }^{13}$ Moreover, switching between the two phases can be triggered externally by applying electrical or laser pulses.
The transition from the amorphous state to the crystalline state typically requires pulses with a duration of hundreds of nanoseconds, although picosecond-order crystallization times have been reported for GST. ${ }^{18,19}$ While PCMs have been 

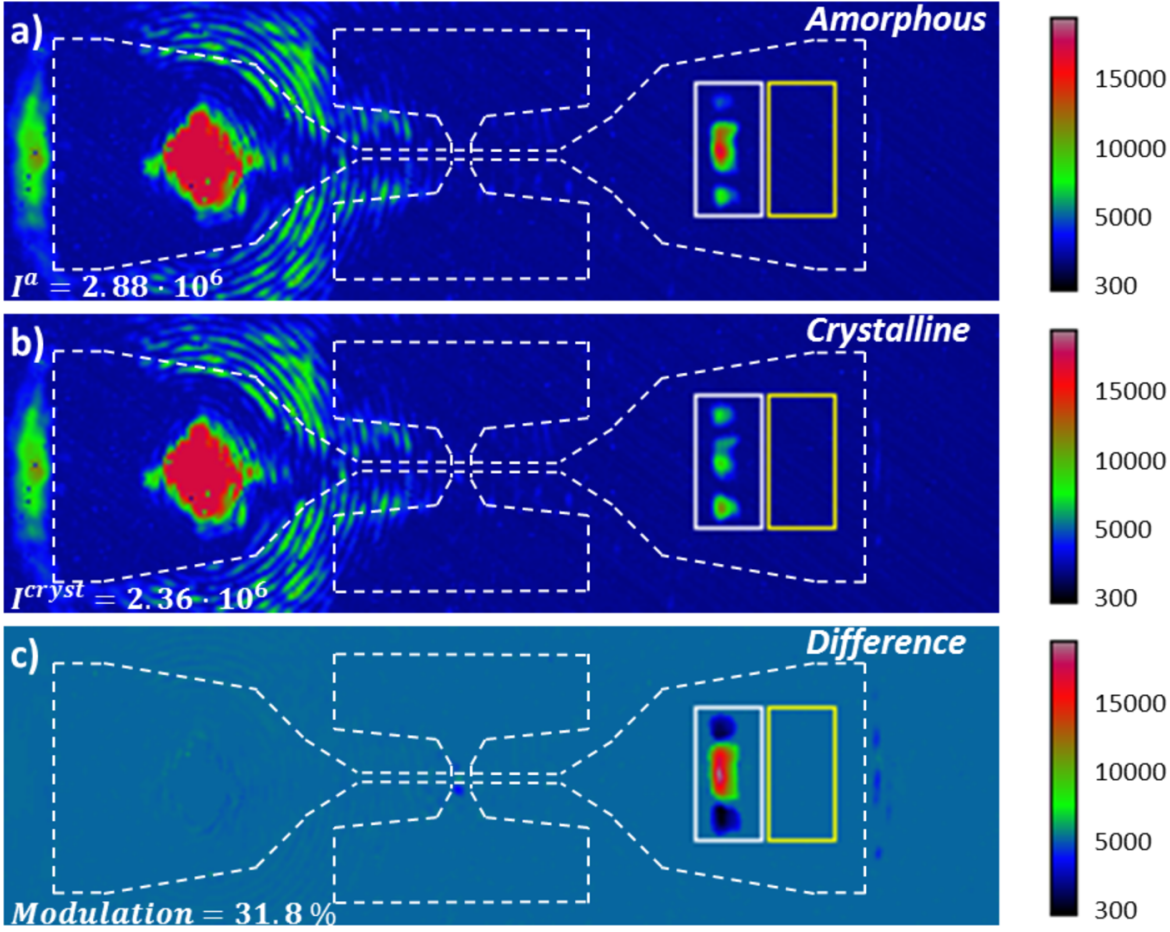

Figure 3. (a, b) Intensity images of the scattered and transmitted laser light at $\lambda=1.5 \mu \mathrm{m}$ as seen by the imaging camera (the dashed lines are an outline of the actual device). The intense spot on the left side corresponds to the focus placed on the in-coupling grating converting propagating light into waveguide modes and SPP modes. The light propagates along the waveguide nearly radiationless and is out-coupled and detected. The intensity at the out-coupling grating changes strongly upon changing the GST phase, leading to a strong differential signal (c).

extensively studied for their application in optical data storage and phase change random access memories (PCRAM), ${ }^{20-22}$ these properties also make them a promising candidate to control and manipulate light in photonic circuits in a nonvolatile manner, an application area that has started to receive an increasing amount of attention. ${ }^{23-25}$

Indeed GST's large change in optical properties, fast switching ability, high cyclability, and ability to retain its structural phase for years without any input energy make it ideal for programming reconfigurable photonic circuits. Herein, we experimentally demonstrate and simulate the use of GST to perform nonvolatile switching of telecom frequency SPPs in plasmonic waveguides.

The change in GST's optical properties upon phase transition can strongly affect the propagation length of the plasmonic mode and hence allows for a change in SPP transmission because of the different attenuation for the amorphous and crystalline phase. Figure 1a illustrates the geometry employed to measure the difference in the propagation length in the $\mathrm{Au} / \mathrm{SiO}_{2} / \mathrm{GST} /$ PMMA/air waveguides for the two phases. Here, the switch is achieved by the phase change in the $30 \mathrm{~nm}$ thin GST layer, which was separated by a $\mathrm{SiO}_{2}$ layer of roughly $150 \mathrm{~nm}$ from $\mathrm{Au}$ and covered by a $2 \mu \mathrm{m}$ thick PMMA layer. The PMMA layer was used to tune the number and properties of the waveguide mode(s) and the $\mathrm{SiO}_{2}$ to thermally isolate the GST from the Au as well as to optimize the sensitivity to the phase transition without substantially sacrificing SPP propagation too much, as explained in the Supporting Information (Figures S1 to S4). The difference of the real part of the mode's propagation constant (cf. Figure S2) enables the selective excitation of the SPP mode using the appropriate spacing of the grooves of the coupling grating in the metal. Additionally, a small groove-like line defect placed $20 \mu \mathrm{m}$ away from the excitation grating (marked by the label A in Figure 1b) is used to probe the SPP intensity, which allows for normalization and elimination of changes in the incoupling efficiency. Similarly, line defects and outcoupling gratings have been engineered at different $L_{\mathrm{G}}$. The resulting transmission is measured by imaging the light at $\lambda=1550 \mathrm{~nm}$ by an InGaAs camera, as shown in Figure 1b. Figure 1c shows the logarithm of the intensity at the output port normalized by the input scattering line defect at point $\mathrm{A}$ obtained from the intensity images at various distances $L_{\mathrm{G}}$. The same measurement is repeated for crystallized GST areas. As shown in the graph in Figure 1c, the intensity along the waveguide decays exponentially for both cases. In the case of crystalline GST, the decay is stronger, which originates from the increased absorption in the GST layer. For distances greater than $80 \mu \mathrm{m}$ the losses are too high and no transmitted intensity could be measured. This strong difference in the SPP waveguide-mode decay length can be used to control optical signals at customized contrast simply by choosing the adequate length of the active region.

The design of our nonvolatile plasmonic switch, sketched in Figure 2a and c, consists of two grating couplers to convert propagating waves into waveguide modes or SPPs at the input port and vice versa at the output port. The two ports are connected by a narrow $(2 \mu \mathrm{m})$ SPP waveguide made of gold, as illustrated in Figure 2b. Additionally, part of the SPP waveguide is covered by the active PCM material. This allows to control the transmitted intensity by changing from the low-loss amorphous phase to the crystalline phase, which has higher propagation losses. The same scheme can be used to electrically drive the phase transition in the GST layer using the tapered design with two electrodes. This design increases the electrical current density at the region where the GST and the Au waveguide cross over, thus providing a hybrid platform to optically or electrically switch SPPs. 
The devices shown in Figure $2 \mathrm{~b}$ have been fabricated using multiple lithographic steps. The metallic pads containing the gratings and waveguide parts have been structured by first employing a positive tone e-beam resist. Subsequently, $30 \mathrm{~nm}$ thick $\mathrm{Ti}$ and then $60 \mathrm{~nm}$ thick Au films are deposited by thermal evaporation. The $\mathrm{Ti}$ layer serves as an adhesion layer and suppresses the coupling of SPPs at the lower interface between the $\mathrm{Au}$ and the $\mathrm{SiO}_{2}$ substrate, which would otherwise contribute to the transmitted intensity. After lifting-off the residual photoresist at the areas unexposed by the electron beam, a top layer of $150 \mathrm{~nm}$ of $\mathrm{SiO}_{2}$ was radio frequency $(\mathrm{RF})$ sputtered from a high-purity target in an Ar atmosphere of $0.5 \mathrm{~Pa}$. The second lithographic step defines the GST strips in the center of the waveguides, where we induce the phase change. The $80 \mathrm{~nm}$ thick GST films were deposited by RF co-sputtering from stoichiometric $\mathrm{GeTe}$ and $\mathrm{Sb}_{2} \mathrm{Te}_{3}$ targets. Energy dispersive X-ray (EDX) measurements performed on GST films that were prepared under the same conditions verified that the composition was equal to the stoichiometric $\mathrm{Ge}_{2} \mathrm{Sb}_{2} \mathrm{Te}_{5}$, with an error smaller than 5 at. \%. Additionally, X-ray diffraction (XRD) measurements confirmed the as-deposited films to be in the amorphous phase. Finally a $20 \mathrm{~nm}$ thick capping layer of $\mathrm{Si}_{3} \mathrm{~N}_{4}$ was deposited by dc sputtering from a $\mathrm{Si}$ target in a reactive $\mathrm{Ar}: \mathrm{N}_{2}$ atmosphere at 0.5 $\mathrm{Pa}$. The $\mathrm{Si}_{3} \mathrm{~N}_{4}$ cap is necessary to protect the GST from oxidation and to confine heat in the structure during the switching process. In a final step, the remaining resist was removed and the whole device was covered with a $2 \mu \mathrm{m}$ layer of PMMA.

The optical micrograph of a device employing a $2 \mu \mathrm{m}$ wide SPP waveguide is shown in Figure $2 b$. The in- and out-coupling gratings have been placed on larger gold pads to reduce the direct transmission of the incident probe laser and to funnel the SPPs to the narrow SPP waveguide. ${ }^{26,27}$ The light-to-SPP conversion can be controlled using the number of grooves and their shape. ${ }^{28}$ Linearly polarized light $(\lambda=1.55 \mu \mathrm{m})$ from a laser diode is focused using a $20 \times$ objective onto the in-coupling grating on the left in Figure 3. The grating periodicity, $G=1 \mu \mathrm{m}$, is designed to couple the incident light, which has no in-plane momentum, into SPPs by fulfilling the condition $k_{\mathrm{SPP}}=2 \pi / i G$, where $i$ is an integer. ${ }^{29}$ The actual value of $k_{\mathrm{SPP}}$ was found by numerically solving the eigenvalue problem for the stratified media using OpenMaXwell. ${ }^{30}$ The out-coupling grating on the right is used to convert the transmitted SPPs into far-field radiation, where it is collected using a $50 \times$ objective and imaged using an InGaAs camera, as shown in Figure 3. The SPP waveguide width is $2 \mu \mathrm{m}$ and its length is $25 \mu \mathrm{m}$, which is smaller than the SPP propagation length for Au at $\lambda=1.55 \mu \mathrm{m}\left(\Lambda_{\mathrm{SPP}} \approx 75 \mu \mathrm{m}\right.$ for $\mathrm{Au}$ embedded in a dielectric slab).

The principle of operation is as follows. A $5 \mu \mathrm{m}$ wide GST strip is structured on top of the SPP waveguide and separated by a $\mathrm{SiO}_{2}$ layer in order to force the SPP to sense the presence of the GST. The thickness of the $\mathrm{SiO}_{2}$ film $(150 \mathrm{~nm})$ is chosen to obtain a strong mode overlap with the $80 \mathrm{~nm}$ GST film, to improve heat trapping in the GST layer, and to prevent chemical reactions between the $\mathrm{Au}$ and the GST. In the initial amorphous state, the film has low absorption and behaves like a dielectric, allowing the SPP to propagate along the waveguide, while in the crystalline state the SPPs are reflected and attenuated due to the strong absorption present in this layer. The structural phase transition in GST is triggered using a control laser $(\lambda=975 \mathrm{~nm})$ focused on the GST area down to a spot size of $4 \mu \mathrm{m}$ using the same objective as the probe laser. The focused laser spot size is larger than the GST-Au crossover area to ensure that the GST filmwhich is directly above the SPP waveguide-completely crystallizes for widths below $4 \mu \mathrm{m}$, as can be seen in the inset of Figure $2 \mathrm{~b}$. By applying single laser pulses $\left(t_{\mathrm{fwhm}}=300 \mathrm{~ns}, t_{\text {rise }}=\right.$ $80 \mathrm{~ns}, P=23 \mathrm{~mW})$, the temperature inside the film increases above the crystallization temperature of the PCM, which for this heating rate is expected to be $T_{\text {cryst }}>625 \mathrm{~K}^{17,31}$ and a polycrystalline region is obtained, corresponding to the brighter area in the inset in image Figure $2 \mathrm{~b}$ due to the increased refractive index and absorption. At $\lambda=975 \mathrm{~nm}$ the absorption of GST is high $(k=1)$ and the penetration depth of the laser at this wavelength is approximately $d_{\text {amor }}=77 \mathrm{~nm}$, which is smaller than the film thickness. Thus, most of the incident laser power will be absorbed by the $80 \mathrm{~nm}$ thick film, which will then crystallize. Although not shown in this work, for a full switching cycle a remorphization process is also present, which is much more difficult to achieve. In this case the penetration depth of the laser in the crystalline GST is $d_{\text {cryst }}=40 \mathrm{~nm}(k=2$ at $\lambda=975 \mathrm{~nm})$, and even more energy will be absorbed. However, much higher temperatures and cooling rates are necessary in order to melt the crystalline phase and quench the resulting liquid state to obtain the amorphous phase, and this could be achieved using the $\mathrm{Au}$ waveguide as a heat-sinking structure to rapidly cool the molten GST.

The transmitted $\lambda=1.55 \mu \mathrm{m}$ light before and after the GST phase change is shown in Figure 3a and b. After irradiating the GST, the transmitted signal is visibly and quantifiably less intense, as shown in Figure $3 b$. To quantify the contrast due to the GST phase change, we integrated the intensity in the rectangular area on top of the out-coupling grating, as indicated by the white rectangle in Figure $3 \mathrm{a}$ and $\mathrm{b}$ for the amorphous phase $I^{\mathrm{a}}$ and after crystallization $I^{\text {crys }}$. The data have been background corrected by measuring the thermal and readout noise in the close vicinity of the grating, as indicated by the yellow rectangle in Figure $3 \mathrm{a}$ and $\mathrm{b}$, resulting in values for $I_{\mathrm{bg}}^{\mathrm{a}}$ and $I_{\mathrm{bg}}^{\text {crys }}$. As the insertion loss of the device depends only on the coupling efficiency of the input grating and is thus independent of the phase of the GST film, these values can be used to calculate the contrast of the device, which is given by

$$
C=\left|\frac{\left(I^{\text {crys }}-I_{\mathrm{bg}}^{\text {crys }}\right)-\left(I^{\mathrm{a}}-I_{\mathrm{bg}}^{\mathrm{a}}\right)}{I^{\mathrm{a}}-I_{\mathrm{bg}}^{\mathrm{a}}}\right|
$$

The strong SPP contrast by the GST phase transition is readily visualized by taking the difference of the camera images as shown in Figure 3c. The majority of the image pixels (nearly) cancels, and a large difference in the intensity is found only at the position of the out-coupling grating, which supports our intention to inhibit SPP propagation by inducing a phase change in the GST layer. In this case the contrast of the device is approximately $31 \%$. The experimental error, obtained by calculating the difference in the average intensity of the same image taken at two different times, is smaller than $0.5 \%$ for both phases.

Using a fluence of $1.8 \mathrm{~W} \mathrm{~m}^{-2}$, the total energy required for the switching process is $6.9 \mathrm{~nJ}$, which is comparable to other plasmonic switches. ${ }^{10}$ Other approaches to switch/modulate SPPs have been shown to need less energy; ${ }^{7-9}$ however these schemes are not nonvolatile and require a continuous external stimulus, thus making it difficult to design reconfigurable plasmonic circuits.

For future modulating devices based on the same scheme it would be necessary to perform several crystallization/reamorphization cycles. Typically for optical data storage applications GST can withstand $10^{5}$ cycles, while for electrical data storage the cyclability is even higher $\left(10^{7}\right.$ cycles $) .{ }^{17}$ Interestingly, the number 
of cycles increases with decreasing energy required to trigger the phase transition. ${ }^{32}$ The GST volumes used in this work are huge $\left(0.08-0.8 \mu \mathrm{m}^{3}\right)$, and we expect cycleabilities smaller than $10^{5}$ cycles. With regard to the maximum frequency achievable by the device a full modulation cycle would include both an amorphous/crystalline and a crystalline/amorphous transition. The minimum time required to trigger these transitions will ultimately limit the modulation speed of the device. In our devices crystallization is achieved using $300 \mathrm{~ns}$ pulses. Reamorphization of GST usually requires fast quench rates on the order of $10^{9} \mathrm{~K} / \mathrm{s}$; thus cooling the material from the melting temperatue $(1000 \mathrm{~K})$ to room temperature $(300 \mathrm{~K})$ would take approximately $700 \mathrm{~ns}$. A full modulation cycle could be achieved in approximately $1 \mu \mathrm{s}$, obtaining a modulation frequency of 1 MHz.

The contrast is based on the fact that the SPPs have significantly different absorption lengths for the two GST phases, which allows one to engineer the contrast. In order to evaluate the influence of the GST area on the performance of the device, the same experiment was repeated using different GST widths $(0.5,1,1.5,2,2.5$, and $3 \mu \mathrm{m})$ for a fixed Au waveguide width of $2 \mu \mathrm{m}$ (Figure 4). For each PCM length the

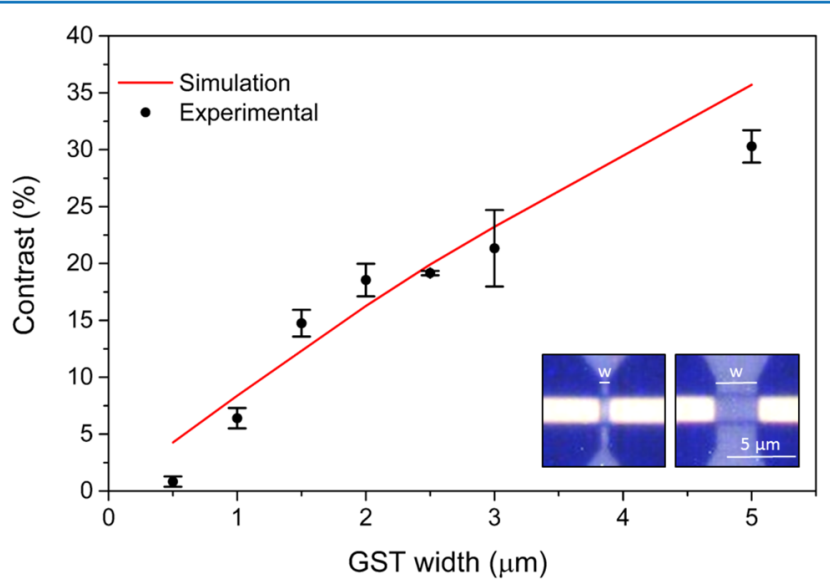

Figure 4. Contrast increases for wider GST strips because GST in the crystalline phase introduces significantly stronger transmission losses compared to its amorphous counterpart.

measurement has been performed on three different devices. The experimental points in Figure 4 indicate the mean value of the contrast, and the error bars its standard deviation. For the wide GST strip $(w=5 \mu \mathrm{m})$, full crystallization could not be achieved due to the spot size of the laser being smaller than the overlap area, obtaining an experimental contrast smaller than the simulated one. As expected, the contrast increases for wider active regions of GST due to a higher interaction length, which leads to larger cumulative absorption of the light propagating in the SPP waveguide mode.

In conclusion, we have demonstrated inhibition of SPP propagation in a $\mathrm{Au} / \mathrm{SiO}_{2}$ interface by exploiting the high contrast in the optical properties of the phase change material $\mathrm{Ge}_{2} \mathrm{Sb}_{2} \mathrm{Te}_{5}$. The attenuation in the transmitted intensity strongly depends on the interaction length between the Au waveguide and the GST film, obtaining a contrast of more than $30 \%$ for sufficiently wide GST strips, using low switching energies to inhibit SPP propagation in a nonvolatile manner. The results demonstrate that the combination of surface plasmon polaritons with phase change materials, such as GST, allows for designing and fabrication of novel active devices such as plasmonic switches or reconfigurable optical circuits.

Finally, we expect that larger contrasts could be achieved either by using larger overlap areas or working at other wavelengths where GST exhibits even higher absorption and contrast, while an improved thermal design could reduce the crystallization time from hundreds of nanoseconds down to the picosecond time scale, thus reducing the switching energy. Moreover, smaller devices that incorporate highly efficient phase change material structures and new switching paradigms, such as coherent phonon excitation, ${ }^{33}$ could present a viable way to reduce the amount of energy deposited in the structure and extend the cycling endurance of the device.

\section{ASSOCIATED CONTENT}

\section{S Supporting Information}

Simulation of the characteristic eigenmodes in the plasmonic waveguide for the armophous and crystalline phases of GST. Influence of the $\mathrm{SiO}_{2}$ and PMMA thicknesses in the propagation constants of the different modes. The Supporting Information is available free of charge on the ACS Publications website at DOI: 10.1021/acsphotonics.5b00050.

\section{AUTHOR INFORMATION}

\section{Corresponding Author}

*E-mail: miquel.rude@icfo.es.

\section{Notes}

The authors declare no competing financial interest.

\section{ACKNOWLEDGMENTS}

We acknowledge financial support from the Spanish Ministry of Economy and Competitiveness (MINECO) and the Fondo Europeo de Desarrollo Regional (FEDER) through grant TEC2013-46168-R, the European Research Council through grant 259196 (PLASMOLIGHT), and Fundació Privada CELLEX.

\section{REFERENCES}

(1) Born, M., Wolf, E. Principles of Optics: Electromagnetic Theory of Propagation, Interference and Diffraction of Light; Cambridge Univ. Press, 1999.

(2) Ozbay, E. Plasmonics: Merging photonics and electronics at nanoscale dimensions. Science 2006, 311, 189-193.

(3) Gramotnev, D. K.; Bozhevolnyi, S. I. Plasmonics beyond the diffraction limit. Nat. Photonics 2010, 4, 83-91.

(4) Schuller, J. A.; Barnard, E. S.; Cai, W.; Jun, Y. C.; White, J. S.; Brongersma, M. L. Plasmonics for extreme light concentration and manipulation. Nat. Mater. 2010, 9, 193-204.

(5) Maier, S. A.; Brongersma, M. L.; Kik, P. G.; Meltzer, S.; Requicha, A. A.; Atwater, H. A. Plasmonics - a route to nanoscale optical devices. Adv. Mater. 2001, 13, 1501-1505.

(6) Barnes, W. L. Surface plasmon-polariton length scales: A route to sub-wavelength optics. J. Opt. A: Pure Appl. Opt. 2006, 8, S87.

(7) Dicken, M. J.; Sweatlock, L. A.; Pacifici, D.; Lezec, H. J.; Bhattacharya, K.; Atwater, H. A. Electrooptic modulation in thin film barium titanate plasmonic interferometers. Nano Lett. 2008, 8, 40484052.

(8) Dionne, J. A.; Diest, K.; Sweatlock, L. A.; Atwater, H. A. PlasMOStor: A metal-oxide-Si field effect plasmonic modulator. Nano Lett. 2009, 9, 897-902.

(9) Pacifici, D.; Lezec, H. J.; Atwater, H. A. All-optical modulation by plasmonic excitation of CdSe quantum dots. Nat. Photonics 2007, 1, 402-406. 
(10) Pala, R. A.; Shimizu, K. T.; Melosh, N. A.; Brongersma, M. L. A nonvolatile plasmonic switch employing photochromic molecules. Nano Lett. 2008, 8, 1506-1510.

(11) Liu, M.; Yin, X. B.; Ulin-Avila, E.; Jeng, B. S.; Zentgraf, T.; Ju, L.; Wang, F.; Zhang, X. A graphene-based broadband optical modulator. Nature 2011, 474 (7349), 64-67.

(12) Lencer, D.; Salinga, M.; Grabowski, B.; Hickel, T.; Neugebauer, J.; Wuttig, M. A map for phase-change materials. Nat. Mater. 2008, 7, 972977.

(13) Shportko, K.; Kremers, S.; Woda, M.; Lencer, D.; Robertson, J.; Wuttig, M. Resonant bonding in crystalline phase-change materials. Nat. Mater. 2008, 7, 653-658.

(14) Huang, B.; Robertson, J. Bonding origin of optical contrast in phase-change memory materials. Phys. Rev. B 2010, 81 (8), 081204.

(15) Welnic, W.; Botti, S.; Reining, L.; Wuttig, M. Origin of the optical contrast in phase-change materials. Phys. Rev. Lett. 2007, 98, 236403.

(16) Robertson, J. Electronic and atomic structure of $\mathrm{Ge}_{2} \mathrm{Sb}_{2} \mathrm{Te}_{5}$ phase change memory material. Thin Solid Films 2007, 515, 7538-7541.

(17) Wuttig, M., Raoux, S., Eds. Phase Change Materials: Science and Applications; Springer Verlag: New York, 2008.

(18) Hegedüs, J.; Elliott, S. R. Microscopic origin of the fast crystallization ability of Ge-Sb-Te phase-change memory materials. Nat. Mater. 2008, 7, 399-405.

(19) Loke, D.; Lee, T. H.; Wang, W. J.; Shi, L. P.; Zhao, R.; Yeo, Y. C.; Chong, T. C.; Elliott, S. R. Breaking the speed limits of phase-change memory. Science 2012, 336 (6088), 1556-1569.

(20) Wuttig, M.; Yamada, N. Phase-change materials for rewriteable data storage. Nat. Mater. 2007, 6, 824-832.

(21) Kolobov, A. V.; Fons, P.; Frenkel, A. I.; Ankudinov, A. L.; Tominaga, J.; Uruga, T. Understanding the phase change mechanism of rewritable optical media. Nat. Mater. 2004, 3, 703-708.

(22) Lankhorst, M. H. R.; Ketelaars, B. W.; Wolters, R. A. M. Low-cost and nanoscale non-volatile memory concept for future silicon chips. Nat. Mater. 2005, 4, 347-352.

(23) Pernice, W. H. P.; Bhaskaran, H. Photonic non-volatile memories using phase change materials. Appl. Phys. Lett. 2012, 101, 171101.

(24) Rudé, M.; Pello, J.; Simpson, R. E.; Osmond, J.; Roelkens, G.; van der Tol, J. J. G. M.; Pruneri, V. Optical switching at $1.55 \mu \mathrm{m}$ in silicon racetrack resonators using phase change materials. Appl. Phys. Lett. 2013, 103, 141119.

(25) Rios, C.; Hosseini, P.; Wright, C. D.; Bhaskaran, H.; Pernice, W. P. On-chip photonic memory elements employing phase-change materials. Adv. Mater. 2014, 26, 1372-1377.

(26) Barnes, W. L.; Dereux, A.; Ebbesen, T. W. Surface plasmon subwavelength optics. Nature 2003, 424, 824-830.

(27) Weeber, J. C.; González, M. U.; Baudrion, A. L.; Dereux, A. Surface plasmon routing along right angle bent metal strips. Appl. Phys. Lett. 2005, 87, 221101.

(28) Renger, J.; Grafström, S.; Eng, L. M. Direct excitation of surface plasmon polaritons in nanopatterned metal surfaces and thin films. Phys. Rev. B 2007, 76, 045431.

(29) Raether, H. Surface Plasmons on Smooth Surfaces; Springer-Verlag: Berlin, 1988 .

(30) OpenMaXwell. http://openmax.ethz.ch.

(31) Orava, J.; Greer, A. L.; Gholipour, B.; Hewak, D. W.; Smith, C. E. Characterization of supercooled liquid $\mathrm{Ge}_{2} \mathrm{Sb}_{2} \mathrm{Te}_{5}$ and its crystallization by ultrafast-heating calorimetry. Nat. Mater. 2012, 11, 279-283.

(32) Raoux, S.; Burr, G. W.; Breitwisch, M. J.; Rettner, C. T.; Chen, Y.; Shelby, R. M.; Salinga, M.; Krebs, D.; Chen, S. H.; Lung, H. L. Phasechange random access memory: A scalable technology. IBM J. Res. Dev. 2008, 52, 465-479.

(33) Makino, K.; Tominaga, J.; Hase, M. Ultrafast optical manipulation of atomic arrangements in chalcogenide alloy memory materials. Opt. Express 2011, 19, 1260-1270. 